\section{COVID-19 Outbreak Associated with Air Conditioning in Restaurant, Guangzhou, China, 2020}

\author{
Ana M. Rule \\ Author affiliation: Johns Hopkins Bloomberg School of Public \\ Health, Baltimore, Maryland, USA
}

DOI: https://doi.org/10.3201/eid2611.202948

To the Editor: In the research letter by J. Lu et al. (1), the authors claim that "The air outlet and the return air inlet for the central air conditioner were located above table C (Figure, panel B)." This sentence does not describe the actual layout depicted in the Figure, in which the air conditioner is located by table $C$ and the exhaust fan is between tables B and D.

Furthermore, the authors do not provide evidence of why "Virus transmission in this outbreak cannot be explained by droplet transmission alone." Their discussion does not mention the possibility that persons move around and may have been infected by touching surfaces, going to the restroom at the same time, or engaging in other close contact.

It is hard to understand how the authors conclude that "... strong airflow from the air conditioner could have propagated droplets from table $C$ to table A, then to table B, and then back to table C." According to the figure, air flows from table $C$ to the exhaust fan (tables B-D). The authors do not provide evidence that the exhaust fan was not working; they ignored its presence. A simple measurement of air flow would answer this question.

The fact that "... none of the staff or other diners in restaurant X were infected" is another indication that the air conditioner was probably working. Also puzzling is the authors' conclusion that "... the smear samples from the air conditioner were all nucleotide negative." This finding is less consistent with aerosol transmission."

The authors' conclusion that "... in this outbreak, droplet transmission was prompted by air-conditioned ventilation" is not supported by the data provided. They further conclude that "The key factor for infection was the direction of the airflow" but do not follow the airflow to the exhaust fan.

\section{Reference}

1. Lu J, Gu J, Li K, Xu C, Su W, Lai Z, et al. COVID-19 outbreak associated with air conditioning in restaurant, Guangzhou, China, 2020. Emerg Infect Dis. 2020;26:1628-31. https://doi.org/10.3201/eid2607.200764
Address for correspondence: Ana M. Rule, Johns Hopkins Bloomberg School of Public Health Department of Environmental Health and Engineering, 615 N. Wolfe St. E6614, Baltimore, MD 21205; USA; email: arule1@jhu.edu

\section{Jianyun Lu, Zhicong Yang}

Author affiliation: Guangzhou Center for Disease Control and Prevention, Guangzhou, China

DOI: https://doi.org/10.3201/eid2611.203774

In Response: We thank Prof. Rule (1) for her comments on our letter (2). We welcome the opportunity to offer additional information on several of the points made.

We wish to explain that although she stated that, "The air outlet and the return air inlet for the central air conditioner were located above table C (Figure, panel B)' does not describe the actual layout depicted in the Figure, in which the air conditioner is located by table $C$ and the exhaust fan is between tables $B$ and $\mathrm{D}^{\prime \prime}$ (1). In fact, the air outlet and the return air inlet for the central air conditioner were located above table C (Figure 1). The central air conditioner is constructed in 2 parts: air outlet and air inlet, indicating no discrepancy between the text and the figure.

We agree that virus transmission in this outbreak could be explained by droplet transmission and the possibility that persons move around, touch surfaces, go to the restroom, or engage in other close contact. We con-

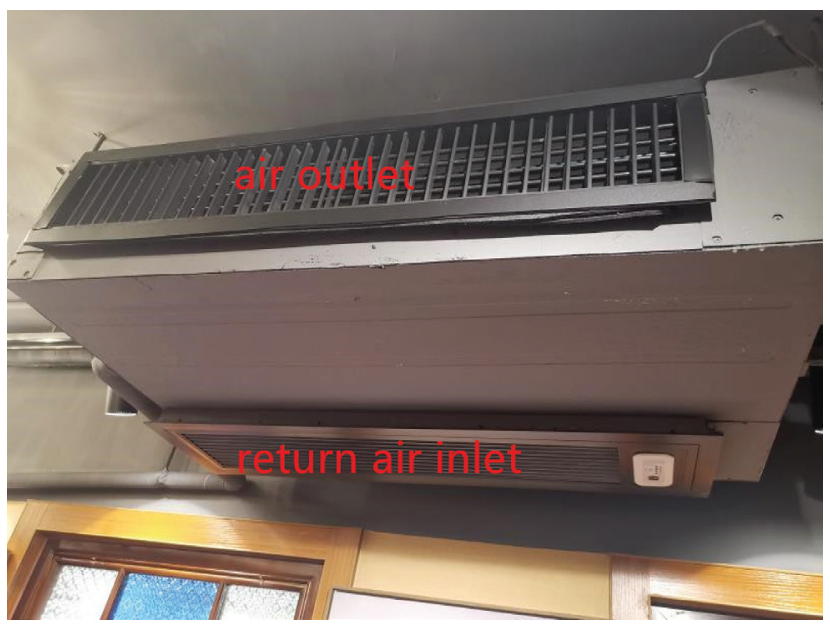

Figure 1. Inlet and outlet of air conditioner described in study of COVID-19 outbreak associated with air conditioning in restaurant, Guangzhou, China, 2020 (2). 